\title{
Modelos de atracción de los y las adolescentes. Contribuciones desde la socialización preventiva de la violencia de género ${ }^{1}$ Modells to attract adolescents. Preventive socialization duties of gender violence
}

\author{
María Padrós Cuxart y Adriana Aubert Simon \\ UNIVERSIDAD DE BARCELONA \\ Patricia Melgar Alcantud \\ UNIVERSIDAD DE GIRONA
}

Resumen

La violencia de género está también presente en las relaciones adolescentes. En España, en el año $2007^{2}$, más de 4.000 chicas menores de 20 años denunciaron a su pareja o ex pareja por malos tratos. Desde la educación, se nos plantea el reto de desarrollar acciones preventivas en sus primeras relaciones.

En la investigación que presentamos hemos analizado los modelos de atracción de los y las adolescentes. Los resultados destacan que los chicos y chicas se están socializando en unas motivaciones y deseos basados en valores tradicionales y desiguales, que se presentan como la causa más profunda sobre la cual se sustenta la violencia de género.

Palabras Clave: violencia de género, modelos de atractivo, socialización preventiva, adolescentes, relaciones afectivas y sexuales.

\section{Summary}

Gender violence is also in relationships among adolescent people. In 2007, more than 4000 Spanish girls under 20 reported their partners or ex-partners for gender violence. Education suggests the challenge of developing preventive measures in their first relationships.

In the research we are presenting now we have analysed attractiveness models of the teenagers. The results show that girls and boys' socialization is based on traditional and unequal motivations and values, which are the basis of gender violence.

KEY WORDS: gender violence, attractiveness models, preventive socialization, adolescents, affective-sexual relationships. 


\section{Introducción}

La violencia de género es una grave problemática social, que va mucho más allá de la violencia doméstica y afecta a mujeres de todas las edades, culturas y perfiles, dándose en los espacios privados, educativos, de trabajo, etc. En los últimos años es especialmente preocupante el aumento de la violencia de género en relaciones afectivas de adolescentes. Los datos del instituto de la mujer ${ }^{3}$ muestran que en los últimos cinco años cerca de 100 mujeres, de entre 16 y 30 años, han sido asesinadas por su pareja o ex parejas. Investigaciones internacionales, como la llevada a cabo por Straus (2004) con población universitaria, ponen de relieve la presencia de esta problemática entre las chicas y chicos jóvenes, y en diferentes tipos de relaciones, tanto estables como esporádicas. En una amplia revisión de investigaciones internacionales sobre la violencia de género, Oliver y Valls (2004) recogen también evidencias de la frecuencia de la violencia de género entre las adolescentes y jóvenes. Sin embargo, las explicaciones de los motivos que llevan a esta situación, a pesar de los avances logrados en la igualdad entre hombres y mujeres, son aún insuficientes y a menudo basadas en falsos mitos, dificultando la prevención y la superación de esta violencia.

Este artículo presenta la investigación "Modelos de atracción de los y las adolescentes. Contribuciones desde la socialización preventiva de la violencia de género", financiada por el Institut Català de les Dones durante el período 2006-2007, y desarrollada en el marco del Grupo de mujeres SAFO, perteneciente al Centro Especial de Investigación en Teorías y Prácticas Superadoras de Desigualdades (CREA), de la Universidad de Barcelona. Entre otras líneas de investigación, el grupo desarrolla desde hace más de diez años trabajos y proyectos sobre violencia de género, algunos de ellos actualmente vigentes en el Plan Nacional $\mathrm{I}+\mathrm{D}+\mathrm{I}^{4}$, y continúa la línea de teorías del amor iniciada por el investigador Jesús Gómez (2004), que incide precisamente en la socialización en unos determinados valores y modelos de atracción, como una de las causas más profundas de la violencia de género.

Gómez, apoyándose en las teorías sociales de autores como Giddens (1995), Beck y Beck-Gernsheim (2001), Mead (1990) o Elster (2002), argumenta el carácter social e interactivo de las motivaciones y los deseos en las relaciones afectivas (Gómez, 2004), y analiza las motivaciones y deseos de las y los adolescentes en la sociedad del riesgo, contando con la voz de las y los adolescentes participantes en la investigación. Con rigor teórico y metodológico, el autor plantea que las emociones y la atracción se fundamentan en aprendizajes sociales: "Si no se hace nada por evitarlo, la raíz de las emociones más íntimas se alimenta de valores tradicionales que incorporamos a través de los procesos de socialización. Tales valores se van introduciendo poco a poco en nuestro interior y conforman la estructura del modelo de atracción que, al haber sido moldeada sin esfuerzos, funciona como si de algo biológico o antropológico se tratara" (Gómez: 2004, 89).

Elena Duque (2006), en la línea de Gómez, se pregunta si los y las jóvenes de nuestra sociedad están aprendiendo para el amor o para la violencia y centra su estudio en las relaciones afectivo-sexuales que se dan en las discotecas. La autora encuadra su investigación en el feminismo internacional actual, que ha superado el análisis del sexismo en los colores de la ropa y los juguetes, para afrontar los problemas actuales del sexismo, como los acosos sexuales en los centros educativos o la intersección del sexismo con el racismo en las identidades juveniles, y trabaja cómo abordarlos desde la participación de todas las mujeres 
(Beck-Gernsheim, Butler y Puigvert, 2003; Aubert y otras, 2004).

La investigación que presentamos se propuso profundizar en la atracción sexual y su vinculación con la violencia de género. $\mathrm{Su}$ principal finalidad ha consistido en analizar los modelos de atracción de los y las adolescentes, visibilizando los valores de las personas que atraen y la relación que puede haber con la violencia de género, y apuntar propuestas de intervención para la prevención de la violencia de género en las edades de inicio de las relaciones afectivosexuales. Los objetivos específicos consistieron en analizar otras investigaciones internacionales en la misma temática; definir los modelos de atracción de los y las adolescentes partiendo de sus propias voces; elaborar orientaciones educativas; difundir los resultados del proyecto.

La investigación desarrolla, pues, cuestiones tan cruciales como: ¿Por qué escoge una adolescente permanecer en una relación, afirmando que sufre y siendo consciente de que no sale beneficiada de ello? ¿Es porqué el amor es ciego y no lo podemos controlar y, simplemente, está enamorada del chico equivocado? Trabajos como el de Smith, White y Holland (2003) subrayan la importancia de las primeras relaciones afectivo-sexuales (por encima de las experiencias familiares en la infancia) respecto al riesgo de padecer, después, violencia de género. Sin embargo, la revisión de las investigaciones ha confirmado un gran vacío en el análisis de los vínculos entre atracción sexual y violencia de género, tanto en la literatura científica como en los programas de prevención y sensibilización dirigidos a los y las jóvenes. Sommers y Surmann (2005) estudian el impacto de la educación sexual, pero no contemplan el análisis de la atracción y del por qué las y los jóvenes escogen un tipo de relaciones y no otras. Epstein y Johnson (2000) estudian la socialización de la sexualidad y conclu- yen que desde los centros educativos, así como desde los medios de comunicación y las políticas públicas, se sigue reforzando un modelo hegemónico de sexualidad que invisibiliza y oprime al resto de identidades sexuales, pero tampoco profundizan en las atracciones hacia unas $u$ otras actitudes y manifestaciones de la identidad sexual.

Pero si las emociones y deseos no son genéticos, ni inevitables, sino que tienen que ver con el proceso social y con cómo nos socializamos para las relaciones afectivas, existe la posibilidad de una socialización que permita desarrollar deseos y motivaciones desvinculadas de la violencia y sea, en este sentido, preventiva de la violencia de género (Oliver y Valls, 2004; Gómez, 2004; Duque, 2006; Valls et. al., 2008). Desde este punto de partida, el proyecto de investigación que se presenta pretende contribuir al análisis de esta socialización y a la definición de formas de actuación de manera transversal en la prevención de la violencia contra las mujeres, contando con todos los agentes educativos.

\section{Metodología}

La metodología utilizada para desarrollar el proyecto ha sido cualitativa de orientación comunicativa crítica (Gómez, J. y otros, 2006), basada en la reflexión y en la intersubjetividad. Parte de las bases teóricas y principios metodológicos validados por la comunidad científica internacional $y$, a diferencia de otros planteamientos, propone la superación del desnivel metodológico entre los sujetos investigados e investigadores, generando conocimiento a través de la creación de un diálogo intersubjetivo. De esta manera evitamos el trato distanciado de la información y posibles interpretaciones erróneas.

En la primera fase, se realizó una revisión de la literatura científica, sobre investigaciones empíricas desarrolladas en el 
ámbito internacional; y materiales y programas educativos sobre estos modelos de atracción. En la realización de esta búsqueda, empleamos bases de datos de publicaciones científicas internacionales, principalmente Sociofile y la ISI Web of Knowledge, centrándonos en la atracción y el deseo en población adolescente y joven, evitando la dispersión hacia elementos complementarios (percepción el amor, influencia de factores culturales en la violencia, formas de violencia, percepción sobre opciones sexuales, etc.).

En la segunda fase del proyecto, desarrollamos el trabajo de campo cualitativo, con el objetivo de recoger las percepciones, interpretaciones y vivencias de las y los menores sobre quiénes atraen y sobre los valores y modelos a los cuales responden estas personas; así como la posible vinculación entre estos modelos y la violencia de género. A su vez, la investigación se centró en reconocer los aspectos sobre los cuales debemos incidir y cómo tenemos que desarrollar actuaciones que potencien modelos de atractivo democráticos.

La muestra del trabajo de campo, estuvo formada por un total de 73 chicos y chicas entre 12 y 18 años de Cataluña, predominantemente de entre 14 y 15 , estudiantes en institutos de educación secundaria de la provincia de Barcelona. La selección de los institutos se llevó a cabo a partir de una relación de centros que habían participado en una campaña educativa de sensibilización sobre la violencia de género durante el curso 2006-2007. De esta manera, se partía de un interés especial por la temática por parte de la dirección del centro. A estos institutos se les hizo llegar la información del proyecto y propuesta de trabajo de campo. La respuesta fue, mayoritariamente, muy receptiva y colaboradora. Se eligieron dos institutos de Barcelona ciudad y cuatro de zonas periféricas. El nivel socio-económico de la población de los institutos selecciona- dos es medio, en un centro el nivel es de clase media-alta y en tres institutos de media-baja. Con algunas excepciones de chicos y chicas inmigrantes, la mayoría de los que participaron en el trabajo de campo no pertenecen a minorías culturales.

Las técnicas de recogida que se aplicaron fueron dos: relatos comunicativos de vida cotidiana y grupos de discusión comunicativos. En total, realizamos 13 grupos de discusión comunicativos ( 5 de chicas, 5 de chicos y 3 mixtos) y 4 relatos comunicativos de vida cotidiana ( 3 chicas, 1 chico).

Los grupos de discusión comunicativos tienen como objetivo llegar a consensos para generar conjuntamente el conocimiento científico y la transformación del contexto, aunque también es posible que éste no se produzca. Estos grupos fueron naturales, es decir, sus componentes tenían un nexo en común, como la participación en alguna actividad conjunta, o la coincidencia en algún espacio físico. En los relatos comunicativos de vida cotidiana la persona participante reflexiona e interpreta sobre su vida cotidiana, centrándose básicamente en el momento actual, y las interpretaciones sirven tanto para proyectar expectativas futuras como para interpretar aspectos del presente o del pasado inmediato. En esta investigación hemos recogido situaciones, reflexiones, recuerdos e interpretaciones de las chicas y chicos, desde una cercanía, basándonos en la importancia y la validez de las interpretaciones de la persona y en el sentido que tienen para ella, en un proceso cooperativo de entendimiento y reflexión orientado a la transformación.

El guión de los relatos y los grupos de discusión comunicativos giró en torno a cuatro grandes temas: definición y construcción de los modelos de atractivos, relaciones afectivo-sexuales y conocimiento de situaciones de violencia de género. Con el objetivo de profundizar en la descripción de detalles y situaciones, en algunos relatos 
comunicativos de vida cotidiana se introdujo en el guión la descripción de situaciones determinadas, sobre las cuales plantear el diálogo y vincularlo a los modelos de atracción. Estas situaciones han estado vinculadas a diferentes ámbitos temáticos en los que los y las adolescentes están de alguna manera implicados.

De acuerdo con el enfoque comunicativo crítico de la investigación, el análisis de las transcripciones de cada una de las técnicas se basó en la identificación de componentes exclusores (suponen barreras o dificultades para las relaciones libres de violencia de género) y transformadores (superan aquellas barreras o dificultades), respecto a cada una de las categorías establecidas: modelos de atracción; interacciones en la construcción social de la atracción; tipos de relaciones; percepciones sobre la violencia de género.

Finalmente, los resultados de la investigación fueron presentados y discutidos en el Fórum contra las violencias de género, celebrado en noviembre de 2007 en Barcelona, tanto a un grupo de treinta estudiantes de secundaria, como a un grupo de profesionales de la educación y otros ámbitos sociales. Esta discusión pública permitió validar los resultados obtenidos y las propuestas de actuación formuladas, fortaleciendo el carácter comunicativo de la investigación.

\section{Análisis e interpretación de los resultados}

Los datos fruto del trabajo de campo nos permiten conocer mejor la realidad que están viviendo muchos y muchas adolescentes en sus relaciones afectivo-sexuales. No hemos pretendido obtener información estadísticamente representativa y generalizable a toda la población adolescente, sino profundizar en aspectos de fondo de sus relaciones y vidas afectivas. Estos aspectos nos proporcionan conocimiento científico a tener en cuenta en nuestras acciones educativas para que chicas y chicos vivan unas relaciones libres de violencia. Hemos profundizado en los tipos de personas que resultan atractivas para los adolescentes, tanto chicas como chicos. Hemos querido incidir en las actitudes que manifiestan las personas que más atraen, y detectar a su vez si algunas de estas personas resultan atractivas cuando tratan mal a las otras. No hemos obviado tampoco las percepciones que los chicos y chicas tienen alrededor de las relaciones afectivo-sexuales, qué valores priorizan en ellas, si incluyen la pasión, el diálogo y la amistad. A continuación, se presentan algunos de los resultados más relevantes del trabajo de campo cualitativo llevado a cabo, introduciendo citas textuales de las chicas y chicos que participaron en la investigación.

\section{1. ¿Quiénes atraen y por qué?}

El trabajo de campo llevado a cabo refleja, como elementos exclusores, que los esquemas más tradicionales, en el sentido de no igualitarios, siguen presentes de forma clara y contundente en las relaciones afectivas y sexuales de las y los adolescentes. Muchas chicas admiten su atracción hacia aquellos chicos que menosprecian a las mujeres, y cuyos rasgos se asocian al modelo tradicional de masculinidad. Las siguientes citas, hablando de los chicos que más éxito tienen, así lo reflejan:

"Los más populares son los más guapos digamos... o los más cabrones" (GM) "Pues que se ha enrollado con muchas tías pero así a su rollo, le va poniendo los cuernos a una, y a otra, pero nadie dice nada" (RF).

"Pues tiene un carácter un poco fuerte, así que es como imponente. (...) va con unos aires así de superioridad, como que él es más que nadie, que es el mejor" (RF). 
Obviamente, esta atracción se traduce, a su vez, en una desvaloración de los chicos que no asumen este papel. Así, existe cierto rechazo por parte de algunas adolescentes entrevistadas hacia los chicos que están por ellas y las tratan bien, e incluso algunos chicos manifiestan conocer el efecto "contraproducente" de una actitud atenta:

"A veces cansa (se ríen). (...) Sí, es tan bueno de esos que dices... también me llegaría a agobiar. (...) Que siempre te dé la razón y todo eso. (...) Un chico tiene que tener su carácter. (...) es que hay tíos que de buenos que son, que parecen tontos" (GMM).

"Si estás muy encima pues no le gustas... en cambio si pasas..." (GM).

De esta manera, el chico que posee valores como sensibilidad y solidaridad, se considera "soso" y poco atractivo. Son los "amigos" y no los chicos de quienes enamorarse, distinguiéndose por un lado al chico "amigo" y, por el otro, al chico "atractivo". Estas conclusiones coinciden con estudios como el de McDaniel (2005), que desvela algunas de las características de los chicos "atractivos": un chico fuerte, divertido, seguro de sí mismo, romántico, excitante, que guste a las amigas y que sea bueno pero no "demasiado".

Sin embargo, ni McDaniel ni la mayoría de estudios sobre la violencia presente en las relaciones amorosas y sexuales de los y las jóvenes no han introducido el por qué la violencia se considera como algo necesario para mantener el poder del agresor o para demostrar el amor y entrega hacia éste (Jonhson y otros, 2005), por qué se tienen estas motivaciones, cómo se generan. Tampoco las adolescentes participantes en los grupos de discusión parecen preguntarse las razones de su atracción por compañeros que las desprecian o las utilizan.

Por otro lado, encontramos que también se mantiene la identificación de la mujer con un objeto al que poseer, con una importancia aún más marcada del físico en los componentes de atracción, así como el desprecio hacia chicas "fáciles":

"Es guapa. Es una guarra. (...) Para liarse sí, pero para salir no. (...) Para un revolcón y ya está. (...) Está todo el día pegada con los tíos. (...) La vas a sobar y tal y no te dirá nunca que no... jsiempre! (se ríen)" (GM).

Los resultados obtenidos muestran también la poca conciencia que las jóvenes entrevistadas tienen de la doble moral, ya que a menudo la sensación de ser atractiva se asocia al número de relaciones mantenidas, extremo que cómo vemos no coincide con las valoraciones realizadas por parte de sus compañeros.

También debemos destacar que existen adolescentes, chicos y chicas, que no siguen los parámetros hegemónicos y tradicionales de atracción y elección. En efecto, desde nuestra investigación también hemos profundizado en aquellas actitudes y valores de respeto y amor que socializan en la atracción hacia las relaciones igualitarias, identificado factores transformadores que llevan a no sólo elegir a quienes tratan con respeto e igualdad, sino también a rechazar a quienes no lo hacen:

"A mí no me gusta que sean cabroncetes conmigo (...) lo veo feo que hagan lo que quieran contigo. Lo veo feo" (GF).

\subsection{Relaciones estables y relaciones espo- rádicas}

Los chicos y las chicas que han participado en el trabajo de campo distinguen claramente entre las características de las relaciones estables y las de las relaciones esporádicas, manifestando diferentes criterios de elección de las personas, según el tipo de relación a mantener. Estas definiciones, que las y los adolescentes hacen de las per- 
sonas que eligen para desarrollar una relación, reflejan de forma muy significativa rasgos vinculados a la doble moral. En cuanto a los chicos, como hemos visto, por un lado eligen a las chicas "fáciles" para "enrollarse" y, por otro, las chicas que gustan para poder tener una relación "seria". Pero en el caso de las chicas, cuando deciden establecer una relación con los chicos considerados "amigos", están buscando una relación estable, más igualitaria y de amistad en comparación con las esporádicas, pero a menudo este énfasis en la ternura va en contraposición de un menor énfasis por la atracción, pasión, etc. En otros casos, las discusiones o la tensión son vividas como una parte inevitable e, incluso, necesaria para mantener "la chispa" o la emoción.

"Es que sino sería muy monótono, ¿no? Siempre bien, las maripositas, todo rosa, sería muy monótono. Yo creo que sí, no sé, yo si tuviera novio me gustaría discutir con él. A ver, no me gustaría pero... (...). Yo creo que está bien que haya roces, si no es que es todo muy bonito todo siempre. $\mathrm{Y}$ te acabarías cansando" (RF).

La atracción se asocia más, a menudo, a las relaciones esporádicas, percibiéndola menos reflexiva y más instintiva, y más asociada a la dominación, el control, poder o incluso agresividad. Lo que "conviene" parece quedar en segundo término $y$, en general, se asume como principio el hecho de no exigir unos determinados valores o respecto.

"Yo a mi forma de pensar. Yo primero para liarme con un chico o algo, primero tengo que conocer. El que más de macarrilla y va de listo y que tiene las tías en bandeja, pues de rollito, y el que es más serio y quiere "mi primera novia y esa va a ser mi mujer", pues de novio. Y el otro pues de rollito" (GF).
Si interpretamos estos resultados a través de las aportaciones de Gómez (2004), identificamos la falsa dicotomía que el autor señala entre bondad y atractivo, entre amor y excitación, entre estabilidad y pasión. Para el autor, el modelo alternativo de relación es el que rompe estas dicotomías y mezcla amor, excitación, bondad y pasión. Si no es así, dice Gómez, estamos condenados y condenadas, desde la adolescencia, a tener que escoger entre relaciones afectivo-sexuales estables y sin pasión, o relaciones excitantes pero vinculadas a la agresividad y la violencia. Debemos decir, también, que en el trabajo de campo aparecen indicios de este modelo alternativo y que consideran que una mayor confianza debe llevar y lleva a un mayor deseo sexual y pasión en la relación.

\subsection{La influencia de las interacciones en la atracción}

Como se ha indicado más arriba, las y los jóvenes entrevistados perciben la atracción como algo que de forma "natural" se da hacia unas personas y no tanto hacia otras. En la siguiente afirmación, por ejemplo, se asume el papel del aprendizaje en la capacidad para mantener unas $\mathrm{u}$ otras relaciones, aunque no en la propia atracción:

"Depende de lo que aprendas, de la familia y de todo. Porque no puedes remediar que te guste, pero sí remediar estar con él" (GF).

Sin embargo, las reflexiones acerca de algunos comentarios, frases, etc. en conversaciones con familiares, vecinos y otras personas muestran que la valoración que el grupo de amistades hace de un modelo de chico o de chica, o de unos comportamientos u otros, por ejemplo, influye significativamente en la construcción de los modelos de atracción y elección afectivo-sexual. En efecto, el deseo de mantener cierta imagen 
delante del grupo de amistades, en función de lo que en ese grupo se esté valorando más, empuja a las y los menores a actuar de determinada manera:

"Cuando está contigo es de una manera..., y después con sus amigos y para hacerse el chulo pues va y es de otra manera... No es tan cariñoso. (...) por quedar bien delante de sus amigos. (...) por la reputación" (GF).

Esta influencia puede enfatizar los valores propios del modelo tradicional, o bien centrar la atención en valores igualitarios:

"Pero también influye la manera de amigos, porque si dicen 'es súper buen pavo y no sé qué, y hace muchas cosas para los demás y tal', tú al final acabas fijándote" (GF).

Como se decía al principio, desde el punto de vista que el amor, el deseo y la atracción sexual son sociales y no instinto, existe la posibilidad de transformar los deseos y la atracción hacia las personas que nos menosprecian o maltratan.

\section{Conclusiones}

Fruto de la revisión bibliográfica llevada a cabo podemos decir que de los estudios sobre el amor y sobre las relaciones afectivas y sexuales que existen en la literatura internacional, escasamente encontramos un análisis en profundidad de las contradicciones que surgen entre lo que se valora para la estabilidad y lo que se desea y asocia a la pasión. Contradicciones que si no resolvemos pueden llevar a las personas a mantener relaciones insatisfactorias e incluso violentas.

Sin embargo, el trabajo de campo desarrollado demuestra la permanencia de los esquemas machistas y violentos en las relaciones y pasiones que mueven a los y las jóvenes que han participado y a sus amistades. Este hecho plantea la necesidad de desarrollar propuestas desde todos los ámbitos de educación social, no solamente hacia las mujeres y los niños y niñas víctimas de la violencia contra las mujeres, sino también hacia la prevención en cada uno de los espacios de atención a la infancia y la juventud, teniendo en cuenta que las relaciones afectivas y sexuales que viven los y las jóvenes (incluyendo todas las relaciones esporádicas) influyen decisivamente en el refuerzo o el rechazo de la violencia o la reproducción del modelo tradicional.

El trabajo de campo nos muestra, además, una falta de reflexión respecto a los motivos que llevan a unas atracciones $u$ otras. Son cuestiones que los chicos y chicas de nuestra muestra no se han planteado anteriormente, que viven de forma bastante instintiva o "irresistible", aunque al reflexionar sobre estas cuestiones, los chi$\cos$ y chicas toman conciencia de la influencia de compañeros, familia, etc., sobre sus gustos y preferencias. Es importante, por lo tanto, que tengan conocimiento de ello y se les ayude a identificar las raíces de sus emociones.

En esta socialización preventiva, en primer lugar, es importante que sepamos identificar los diferentes canales (familia, educación, medios de comunicación) desde los cuales se potencian estos modelos de atracción. En segundo lugar, se deben destacar los valores que se transmiten y sus consecuencias. Finalmente, este análisis debe ir orientado a la potenciación del modelo alternativo de atracción-elección en base a "no eliminar la pasión y el deseo" en él, dotando de atractivo los valores transformadores.

Como ya se ha dicho, el punto de partida de la investigación que hemos presentado es que tanto el amor como la atracción sexual son construcciones sociales y no hechos biológicos, instintivos y 
no controlables. Esto implica la posibilidad de cambiar y transformar la atracción que se siente hacia las personas que nos tratan mal para poder desarrollar relaciones plenamente satisfactorias y apasionadas, sean esporádicas o estables. La clave está, entonces, en la reflexión y diálogo alrededor de estos modelos de atracción, con el fin de transformarlos y liberarlos de la identificación entre deseo y violencia. Para transformar las situaciones de desigualdad en el amor es imprescindible contemplar la posibilidad de diálogo y construcción social a través de la interacción y la reflexión, por lo que el amor ya no se puede considerar algo que nos ocurre fuera de nuestras intenciones y de nuestras actuaciones.

En definitiva, las propuestas que se han formulado como resultado del proyecto de investigación se concretan en tres tipos de acciones a desarrollar en cualquier espacio educativo: 1) informar a través del diálogo a las y los menores sobre aquellas causas de la violencia de género con datos de calidad y científicamente corroborados; 2) reflexionar sobre aspectos como la libertad sexual o el hecho que las relaciones esporádicas que se basan en el modelo de atracciónelección tradicional también socializan en la violencia de género; 3) analizar e identificar los distintos modelos de atractivo que se esconden en sus relaciones.

Las prácticas educativas de socialización preventiva deben orientarse a analizar con los chicos y chicas adolescentes situaciones cotidianas relacionadas con sus relaciones afectivo-sexuales para que puedan reflexionar e identificar cuáles son los modelos de atractivo y las características de estos modelos, destacando si esta atracción dirige la pasión hacia el poder y hacia actitudes no igualitarias, que pueden derivar en una relación de violencia de género, o bien si potencia la atracción por actitudes de respeto, igualdad y amor.

\section{Referencias bibliográficas}

Aubert, A. y otras (2004): Dialogar y transformar. Pedagogía crítica del siglo XXI. Barcelona, Graó.

Beck, U. (1998): La sociedad del riesgo. Barcelona, Paidós.

Beck, U. y Beck-Gernsheim, E. (2001): El normal caos del amor. Barcelona, El Roure.

Beck-Gernsheim, E.; Butler, J. y Puigvert, L. (2003): Women and Social Transformation. Peter Lang Publishing, Nueva York.

Conselleria de Familia, Xuventude, deporte e voluntariado (2004): A violencia contra as mulleres en Galicia.

Duque, E. (2006): Aprendiendo para el amor o para la violencia. Las relaciones en las discotecas. Barcelona, El Roure.

Elster, J. (2002): Alquimias de la mente. La racionalidad y las emociones. Barcelona, Paidós.

Epstein, D. y Johnson, R. (2000): Sexualidades e institución escolar. Madrid, Morata.

Giddens, A. (1995): La transformación de la intimidad. Madrid, Cátedra.

Gómez, J. (2004): El amor en la sociedad del riesgo. Una tentativa educativa. Barcelona, El Roure.

Gómez Alonso, J. y otros (2006): Metodología comunicativa crítica. Barcelona, El Roure.

Johnson, S. y otros (2005): "I Know What Love Means. Gender-based Violence in the Lives of Urban Adolescents". Journal of Women's Health, 14, pp. 172-179.

McDaniel, A. (2005): "Young Women's Dating Behavior: Why/Why Not Date a Nice Guy?". Sex Roles: A Journal of Research, 53, pp. 347-359.

Mead, G. H. (1990): Espíritu, Persona y Sociedad. México, Paidós.

Oliver, E. y Valls, R. (2004): Violencia de género. Investigaciones sobre quiénes, por qué y cómo superarla. Barcelona, El Roure.

Puigvert, L. (2001): Las otras mujeres. Barcelona, El Roure.

Smith, P. H. y otros (2003): "A Longitudinal Perspective on Dating Violence Among Adolescent and College-Age Women". American Journal of Public Health, 93, pp. 104-110. 
Somers, C. y Surmann, T. (2005): "Sources and timing of sex education: relations with American adolescent sexual attitudes and behavior". Educational Review, 57, pp. 37-54.

Straus, M. (2004): "Prevalence of Violence Against Dating Partners by Male and Female University Students Worldwide". Violence Against Women, 10, pp. 790-811.

Valls, R.; Puigvert, L. y Duque, E. (2008): "Gender Violence Among Teenagers: Socialization and Prevention". Violence Against Women, 14 (7), pp. 759-785.

\section{Notas}

${ }^{1}$ Investigación financiada por el Institut Català de les Dones. Ayudas a trabajos de investigación sobre las mujeres. Núm. de referencia: U-44/o6. Investigadora principal: Dra. María Padrós.

${ }^{2}$ Instituto de la Mujer: http://www.mtas.es/mujer/mujeres/cifras/violencia/denuncias tablas.ht m (consultada en mayo de 2008).

${ }^{3}$ Instituto de la Mujer. http://www.mtas.es/mujer/mujeres/cifras/tablas/W807.XLS (consultada en mayo de 2008).
${ }^{4}$ Ver más información en: http://www.pcb.ub.es/ crea/es/gdona_es.htm y http://www.pcb.ub.es/ crea/es/violenciag es.htm

${ }^{5}$ Códigos: G: Grupo de discusión comunicativo. R: Relato comunicativo de vida cotidiana. F: $\mathrm{Mu}-$ jer. M: Hombre. MM: Mixto.

DiRECCIÓN DE LAS AUTORAs: María Padrós Cuxart. Departamento de Didáctica y Organización Educativa. Universidad de Barcelona. Campus Mundet, Edificio Llevant, Passeig Vall d'Hebron, 171, o8035 Barcelona.

Correo electrónico: mariapadros@ub.edu

Fecha de recepción del artículo: 21. VII.2008

Fecha de aceptación definitiva: 30.IX.2009

COMO CITAR ESTE ARTÍCULO:

Aubert Simon, A.; Melgar Alcantud, P. y Padrós Cuxart, M. (2010): "Modelos de atracción de los y las adolescentes. Contribuciones desde la socialización preventiva de la violencia de género". Pedagogía Social. Revista Interuniversitaria, 17, pp. 73-82. 